\title{
Cerebellar fits in children with Chiari I malformation
}

\author{
Aditya Pandey, M.D., Shenandoah Robinson, M.D., and Alan R. Cohen, M.D. \\ Division of Pediatric Neurological Surgery, Rainbow Babies and Children's Hospital, Research \\ Institute of University Hospitals of Cleveland, Case Western Reserve University School of Medicine, \\ Cleveland, Ohio
}

\begin{abstract}
Object. The authors describe a series of children with Chiari I malformation who presented with fulminating symptoms of "cerebellar fits" characterized by drop attacks with or without deterioration of consciousness, opisthotonic posturing, and varying degrees of respiratory compromise.

Methods. A retrospective analysis was undertaken of the medical records of 47 consecutive patients undergoing surgery for symptomatic Chiari I malformations at Rainbow Babies and Children's Hospital.

Thirteen $(28 \%)$ of the 47 patients presented with complaints consistent with cerebellar fits. Before the correct diagnosis was made, nine $(69 \%)$ of the 13 children had previously undergone evaluation with electroencephalography and/or electrocardicography and Holter monitoring because of suspected cortical epilepsy or cardiogenic syncope. In each of the 13 children magnetic resonance imaging demonstrated pegged cerebellar tonsils herniated below the foramen magnum. A deep indentation or blanched discoloration of the cerebellar tonsils was noted in five (38\%) of these 13 patients at the time of surgery. Of patients with symptomatic Chiari I malformations, the mean degree of tonsillar herniation was significantly less for those in whom cerebellar fits occurred than those in whom they were absent $(8.8$ $\mathrm{mm}$ and $13.9 \mathrm{~mm}$, respectively; $\mathrm{p}=0.007)$. In only one of the patients with cerebellar fits was a syrinx present, and this was a small focal lower thoracic collection. Spells resolved after surgery in all patients who presented with cerebellar fits.

Conclusions. Cerebellar fits may mimic other disorders such as cardiogenic syncope and epileptic seizures. The correct diagnosis may be delayed or the conditions may be misdiagnosed by those who fail to consider Chiari I malformation as a cause of drop attacks, abnormal extensor posturing, and apneic spells in children. The response to decompressive surgery in these patients is gratifying.
\end{abstract}

\section{KeY WORDS • Chiari malformation • cerebellum • cerebellar fits • epilepsy • syncope}

The Chiari I malformation is characterized by downward displacement of the cerebellar tonsils through the foramen magnum into the cervical spinal canal. ${ }^{1}$ Clinical manifestations are variable and are usually related to direct compression of neural structures and/or disturbance of cerebrospinal fluid circulation., ${ }^{3,6,9,11-14}$ The most common presenting symptom in patients with Chiari I malformation is pain, and the presenting signs are diverse and are usually related to brainstem or cranial nerve dysfunction, spinal cord dysfunction, or cerebellar dysfunction. ${ }^{7}$ With the widespread use of MR imaging, asymptomatic Chiari I malformation is being diagnosed with increased frequency. ${ }^{10,15}$

Occasionally patients with Chiari I malformation present with fulminating symptoms. The present report concerns a group of children with Chiari I malformation who presented with drop attacks, extensor posturing, or varying degrees of respiratory compromise. This presentation bears similarity to the "cerebellar fits" first described by Jackson in 1871 and reported by him and others to occur as the result of mass effect from cerebellar tumors. ${ }^{8}$

Abbreviations used in this paper: $\mathrm{ECG}=$ electrocardicography; $\mathrm{EEG}=$ electroencephalography; $\mathrm{MR}=$ magnetic resonance.
Patients with Chiari I malformation by definition have chronic cerebellar tonsillar herniation, and fulminating presentations presumably reflect medullary compression as the result of crowding at the craniovertebral junction.

The relationship between cerebellar fits and Chiari I malformation has not been systematically investigated. In reviewing our series of pediatric patients with Chiari I malformation, we were surprised by the frequency of drop attacks as a presenting sign. The diagnosis of Chiari I malformation had often been overlooked in the initial evaluation, and children had frequently undergone EEG, ECG, and Holter monitoring for the detection of epileptic seizures or cardiogenic syncope. The purpose of the present study was to analyze the clinical and radiographic findings in a population of children presenting with cerebellar fits and Chiari I malformation and to discuss the outcome following decompressive surgery.

\section{CLINICAL MATERIAL AND METHODS}

Between 1994 and 2000, 47 consecutive children underwent surgical decompression of the craniovertebral junction for symptomatic cerebellar tonsillar herniation at Rainbow Babies and Children's Hospital. The most common symptom was incapacitating headache, usually occi- 
pital or cervical in location. Other presenting symptoms included irritability (in preverbal patients), weakness, clumsiness, ataxia, and sensory abnormalities. The presence of significant syringomyelia was considered an indication for surgery, although all patients with spinal cord cavitation also had other symptoms or signs (for example, headache or progressive scoliosis). Patients with signs of acute brainstem dysfunction such as drop attacks, abnormal posturing, and respiratory abnormalities were considered to have cerebellar fits (see the Results section). Patients with mild headache and a normal neurological status were managed without operation. Patients with incidental Chiari malformations likewise did not undergo surgery.

A retrospective review of the medical records, MR images, and operative reports was conducted. There were 26 male and 21 female patients. The mean age was 8.5 years. The diagnosis of Chiari I malformation was made only if MR imaging demonstrated that the cerebellar tonsils were pointed and displaced $5 \mathrm{~mm}$ or more below the foramen magnum. The surgical procedure consisted of a limited suboccipital craniectomy to expand the foramen magnum, C-1 laminectomy, and duraplasty. It was rarely necessary to extend the laminectomy below $\mathrm{C}-2$, but in all cases the osseous and dural decompressive procedure was performed inferior to the level of tonsillar descent.

\section{RESULTS}

Of the 47 children with symptomatic cerebellar tonsillar herniation (Chiari I malformation) who underwent craniovertebral decompression, 13 (28\%) had presented with drop attacks, abnormal extensor posturing, and varying degrees of respiratory compromise. These 13 patients with so-called cerebellar fits consisted of seven males and six females who ranged in age from 2 months to 16 years (mean age of 6.7 years). Of the remaining 34 symptomatic patients in whom cerberellar fits were not present, 19 were male and 15 were female, and their ages ranged from 2 to 16 years (mean age 9.6 years).

In the 13 children presenting with cerebellar fits, the duration of symptoms prior to diagnosis ranged from 1 week to 72 months (mean duration 12.1 months). Drop attacks consisted of paroxysmal loss of postural tone usually associated with loss of consciousness, extensor posturing, and apnea. At presentation four of the 13 children had experienced a single episode, whereas the majority had suffered multiple episodes.

Nine of the 13 children with cerebellar fits had undergone diagnostic studies for presumed epileptic seizures or cardiogenic syncope prior to investigation with MR imaging. Eight children were evaluated with EEG. The EEG findings were normal in seven and showed right posterotemporooccipital slowing in one. Three patients were evaluated with ECG and Holter monitoring, which showed normal findings in two and abnormal findings in one (irregular cardiac rhythm).

The extent of tonsillar descent was smaller in patients with cerebellar fits than those with other symptoms. The mean magnitude of tonsillar herniation was $8.7 \mathrm{~mm}$ below the foramen magnum in patients with cerebellar fits, whereas it was $13.8 \mathrm{~mm}$ in those the patients with symptomatic Chiari I malformation without cerebellar fits $(\mathrm{p}=$
0.007 , Student two-tailed t test). Syringomyelia was present in one $(8 \%)$ of the 13 patients with cerebellar fits and consisted of a small focal cavitation of the lower thoracic spine. Of the 34 patients without cerebellar fits, syringomyelia was present in seven $(21 \%)$. No patient in this series had hydrocephalus.

Blanched discoloration of the tonsils or a deep indentation from herniation was noted intraoperatively in five (38\%) of the 13 patients with cerebellar fits. In each of the patients presenting with cerebellar fits, symptoms resolved following surgery; the mean duration of the followup period was 2.5 years.

\section{ILLUSTRATIVE CASES}

\section{Case 1}

This 2.5-year-old girl presented with a 6-month history of spells characterized by loss of consciousness typically lasting 4 minutes, tonic posturing, drop attacks, and apnea. She had a 2-month history of episodic gagging and emesis. Her history was remarkable for complex partial seizures since age 3 months, which consisted of lip smacking and staring spells, as well as hyperactivity and developmental delay. Neurological examination revealed that the patient's speech was incoherent. Results of EEG were normal. Magnetic resonance imaging demonstrated a tight foramen magnum with pointed cerebellar tonsils herniated $10 \mathrm{~mm}$ below the foramen magnum (Fig. 1 left). At surgery, the tips of the tonsils were extremely pale due to chronic compression (Fig. 1 right). Following decompressive surgery, she has continued to experience complex partial seizures but no further drop attacks, loss of consciousness, apnea, gagging, or emesis.

\section{Case 2}

This 3-year-old boy presented with a 1-year history of drop attacks during which he would stiffen and fall suddenly to the ground but not lose consciousness. Each

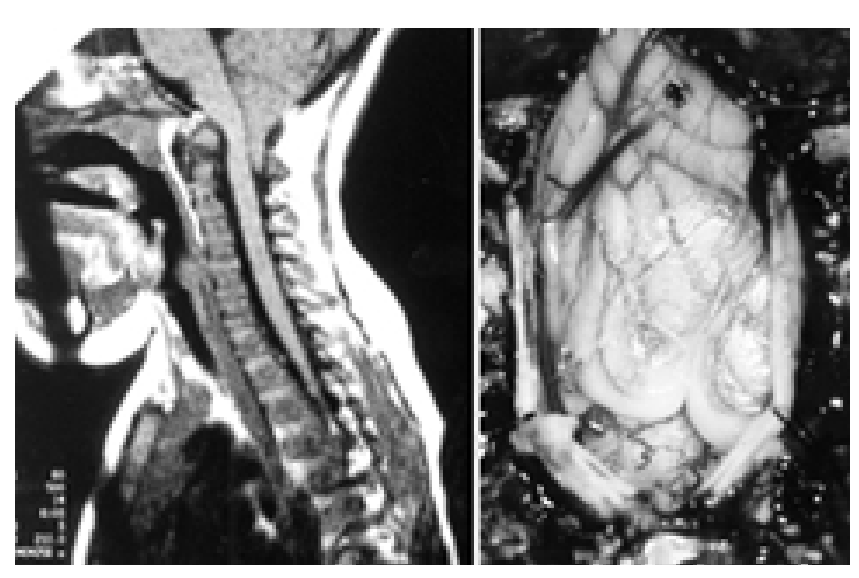

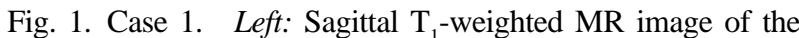
brain. Note herniation of the cerebellar tonsils to the level of C-2, as well as crowding at the foramen magnum. Right: Intraoperative photograph obtained after $\mathrm{C}-1$ and partial C-2 laminectomy and durotomy. Note the pallor at the caudal aspect of the cerebellar tonsils bilaterally. 
episode lasted less than a minute. He complained of posterior neck pain. History was remarkable for generalized seizures in infancy that resolved by 1 year of age. Examination was remarkable for demonstrating left lower-extremity weakness and gait ataxia. Results of EEG were normal. Electrocardiography and 24-hour Holter monitoring were performed prior to reaching the diagnosis of Chiari I malformation. These studies demonstrated normal findings. Magnetic resonance imaging revealed cerebellar tonsillar herniation and crowding of the foramen magnum (Fig. 2). Following surgery, he has experienced no further drop attacks and his strength and gait have improved.

\section{Case 3}

This 4-year-old boy presented with a 1-month history of episodes of extensor posturing with staring and apnea. He complained of headaches exacerbated by the Valsalva maneuver. He had suffered two clonic seizures during his 1st year of life but none since. Examination revealed truncal ataxia. Results of EEG were normal. The diagnosis of Chiari I malformation was made following MR imaging. Postoperatively, he has suffered no further spells and his gait has improved.

\section{DISCUSSION}

Jackson ${ }^{8}$ coined the term "cerebellar fits" in 1871 to describe tetanus-like episodes that occurred in a 5-year-old boy with a midline cerebellar tumor (Fig. 3). The first description of convulsive seizures associated with a cerebellar lesion was reported by Wurffbain in 1691, who described head retraction and opisthotonos in association with a cerebellar tumor. ${ }^{5}$ Cushing $^{2}$ recognized that a pressure cone across the foramen magnum could result in sudden death.

Cerebellar fits have been discussed infrequently in the recent neurosurgical literature. These paroxysmal spells are characterized by drop attacks with or without loss of consciousness, abnormal extensor posturing, and varying degrees of respiratory compromise. Presumably, cerebellar fits were seen more commonly in the era before mod-

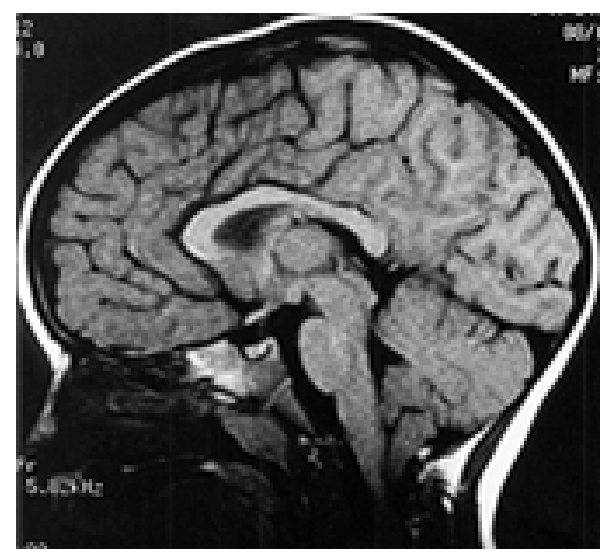

Fig. 2. Case 2. Sagittal $\mathrm{T}_{1}$-weighted MR image of the brain. This child had experienced frequent drop attacks that resolved following surgical decompression of the craniovertebral junction.

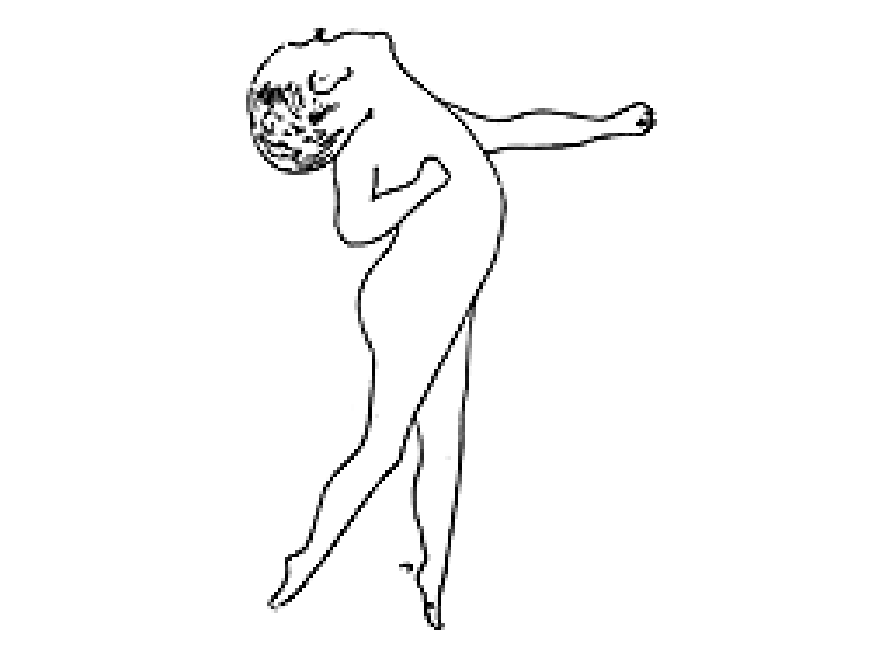

Fig. 3. Drawing by Sir Stephen Mackenzie of a child experiencing a tetanus-like seizure. The drawing was included in Hughlings Jackson's case report of a cerebellar tumor in which he coined the term "cerebellar fits."

ern neuroimaging, when patients with large posterior fossa tumors would present in extremis with cerebellar tonsillar herniation.

Cerebellar fits are not limited to patients with cerebellar tumors, arising likewise in patients with conditions in which there is tonsillar herniation. In our series of children with symptomatic Chiari I malformation, more than $25 \%$ experienced paroxysmal drop spells that could be characterized as cerebellar fits. It is important to remember that children with Chiari I malformation can present with drop attacks, abnormal posturing, and apneic spells. Failure to recognize this association can lead to a delay in diagnosis or even misdiagnosis. Chiari I malformation-related cerebellar fits in children are frequently misdiagnosed initially as epileptic seizures or cardiogenic syncope, and patients have often undergone evaluation with EEG and/or ECG and Holter monitoring before the diagnosis of Chiari I malformation is confirmed on MR imaging.

It is noteworthy that three of our patients with cerebellar fits also had a history of epileptic seizures. Cerebral cortical abnormalities are not generally considered to be part of Chiari I malformation, and we are not sure if this association is significant. The occurrence of true epileptic seizures associated with Chiari I malformation (primarily complex partial fits or paroxysmal abnormalities referable to the frontal or temporal lobes) has been reported previously. ${ }^{4}$ The course of epilepsy in these affected patients is usually benign. Although some patients with Chiari I malformation may have epilepsy, the major finding in our study was that the tonic posturing seen in Chiari I malformation patients with cerebellar fits can be mistaken for epilepsy and lead to a delay in diagnosis.

In children with cerebellar fits and Chiari I malformation the presentation is an extreme manifestation of brainstem dysfunction. The most feared consequences of cerebellar fits in this population are respiratory arrest and sudden death. This outcome may be avoided by early diagnosis. Thus, it is important to remember that cerebellar fits in patients with Chiari I malformation can mimic 


\section{A. Pandey, S. Robinson, and A. R. Cohen}

other paroxysmal events such as epilepsy and cardiogenic syncope. The diagnosis of tonsillar herniation can be confirmed using MR imaging. The response of these patients to decompressive surgery is gratifying.

\section{References}

1. Bindal AK, Dunsker SB, Tew JM Jr: Chiari I malformation: classification and management. Neurosurgery 37:1069-1074, 1995

2. Cushing, H: Some experimental and clinical observations concerning states of increased intracranial tension. Am J Med Sci 124:375-400, 1902

3. Dyste GN, Menezes AH: Presentation and management of pediatric Chiari malformations without myelodysplasia. Neurosurgery 23:589-597, 1988

4. Elia M, Biondi R, Sofia V, et al: Seizures in Chiari I malformation: a clinical and electroencephalographic study. J Child Neurol 14:446-450, 1999

5. Fulton JF: A case of cerebellar tumor with seizures of head retraction described by Wurffbain in 1691. J Nerv Ment Dis 70:577-583, 1929

6. Heiss JD, Patronas N, DeVroom HL, et al: Elucidating the pathophysiology of syringomyelia. J Neurosurg 91:553-562, 1999

7. Iskandar BJ, Oakes WJ: Chiari malformations, in Albright AL, Pollack IF, Adelson PD (eds): Principles and Practice of Pediatric Neurosurgery. New York: Thieme, 1999, pp 165-187

8. Jackson JH: Case of tumour of the middle lobe of the cerebellum-cerebellar paralysis with rigidity (cerebellar attitude) occasional tetanus-like seizures (1871). Brain 29:425-440, 1906
9. Mclone DG: The biological resolution of malformations of the central nervous system. Neurosurgery 43:1375-1381, 1998

10. Meadows J, Kraut M, Guarnieri M, et al: Asymptomatic Chiari Type I malformations identified on magnetic resonance imaging. J Neurosurg 92:920-926, 2000

11. Milhorat TH, Chou MW, Trinidad EM, et al: Chiari I malformation redefined: clinical and radiographic findings for 364 symptomatic patients. Neurosurgery 44:1005-1017, 1999

12. Oldfield EH, Muraszko K, Shawker TH, et al: Pathophysiology of syringomyelia associated with Chiari I malformation of the cerebellar tonsils. Implications for diagnosis and treatment. $\mathbf{J}$ Neurosurg 80:3-15, 1994

13. Park JK, Gleason PL, Madsen JR, et al: Presentation and management of Chiari I malformation in children. Pediatr Neurosurg 26:190-196, 1997

14. Rekate HL, Nadkarni TD, Teaford PA, et al: Brainstem dysfunction in Chiari malformation presenting as profound hypoglycemia: presentation of four cases, review of the literature, and conjecture as to mechanism. Neurosurgery 45:386-391, 1999

15. Wu YW, Chin CT, Chan KM, et al: Pediatric Chiari I malformations: do clinical and radiologic features correlate? Neurology 53:1271-1276, 1999

Manuscript received June 7, 2001.

Accepted in final form June 29, 2001.

Address reprint requests to: Alan R. Cohen, M.D., Division of Pediatric Neurosurgery, Rainbow Babies and Children's Hospital, B-501, 11100 Euclid Avenue, Cleveland, Ohio 44106. email: alan.cohen@uhhs.com. 